\title{
UN PROBLEMA PLANTEADO COMO ACTIVIDAD DE INVESTIGACIÓN: ESTUDIO DE LAS POSIBLES TRAYECTORIAS PARA EL LANZAMIENTO EFECTIVO DE UN TIRO LIBRE DE BALONCESTO
}

\author{
MARTÍNEZ PONS, JOSÉ ANTONIO \\ IES La Lagunas. Rivas Vaciamadrid. \\ Departamento de Química Analítica e Ingeniería Química. Universidad de Alcalá de Henares.
}

\section{SUMMARY}

Although students learn kinematics from their first contact with physics, for a series of reasons preconceptions and conceptual and methodological mistakes accumulate, hindering progress.

We propose a simple and motivating exercise that will not only improve the understanding of elementary equations of kinematics, but also detect and facilitate the correction of such mistakes.

\section{INTRODUCCIÓN}

Normalmente la enseñanza de la física elemental suele hacerse de modo historicista y repetitivo (Furió y Guisasola, 1993; Gutiérrez, 1993; Martínez Aznar, 1996; Martínez Pons, 1995), de modo que, en el esquema tradicional, la cinemática se ve y se vuelve a ver desde la antigua EGB hasta los primeros años de facultad. Cada vez se intenta profundizar más, pero, puesto que muchas veces los primeros conceptos se han aprendido mal, la comprensión se hace difícil, insistiéndose más en el formalismo matemático que en el fenómeno físico real.

La experiencia personal del autor, contrastada con la de otros profesionales (Gutiérrez, 1993), indica que resolver un problema, que en general es más un ejercicio que un auténtico problema, significa «dar con la fórmula adecuada», probablemente enmarcada en rojo en el libro de texto. Poco importa el porqué de dicha fórmula; importa menos su campo de aplicabilidad y muchísimo menos el sentido, ya de la misma fórmula, ya del resul- tado de aplicarla, sobre todo si este último se ha obtenido con calculadora, infalible por definición. Como mucho, se acepta que es error grave no escribir las unidades (que éstas se comprendan es otra cosa). Véase la mayoría de textos de física de $\mathrm{COU}$ y en general de niveles previos a la universidad, que no se citan por razones obvias.

En el campo concreto de la cinemática suelen quedar confusos y entremezclados conceptos fundamentales como los de trayectoria, posición y distancia recorrida. Como consecuencia, las ecuaciones del movimiento no se comprenden en su profundidad y se aplican de modo incorrecto. El papel de los parámetros iniciales del movimiento tampoco queda claro. Las ecuaciones se interpretan como «espacio», nunca como «posición», y el concepto de sistema referencial en la mayoria de casos simplemente se deja de lado. En un problema de lanzamiento de un objeto hacia arriba, por ejemplo, es típico dividir el problema en dos: en el primero, el objeto sube 
y «la gravedad es negativa»; en el segundo, el objeto baja y «la gravedad es positiva» (Martínez Pons, 1995).

Aquí se propone un trabajo que, sin salir de la línea clásica, obliga al alumno a plantearse, desde un punto de vista físico, un problema con el que se tropieza en su vida cotidiana, ya como actor, ya como espectador, y a comprobar que la física no está tan alejada de la realidad.

\section{OBJETO DE LA ACTIVIDAD}

La experiencia aquí descrita se llevó a cabo con estudiantes de COU, a los que se había practicado a principios de curso una prueba de nivel consistente en una batería de preguntas abiertas de respuesta breve y dos problemas algo más elaborados. Las repuestas parecían indicar la existencia de las confusiones antes citadas, una elevada tendencia a la aplicación de fórmulas estereotipadas en la resolución de los ejercicios y un nivel mínimo de razonamiento. Tampoco aparecía la crítica de las condiciones previas del problema, ni de los propios resultados, ni de la verosomilitud del modelo.

Se trataba de buscar una actividad que, además de confirmar la impresión inicial, sirviera, primero, para que los alumnos se autoconvencieran de que su modo de trabajo no era el más adecuado; segundo, para que aclararan sus ideas al respecto; y, tercero, para que buscaran remedio con la ayuda del profesor.

Se decidió que la actividad debía:

1)Ser altamente motivadora. En este sentido el mundo del deporte podía aportar multitud de ejemplos.

2)No implicar un aparato matemático fuera del alcance de los estudiantes.

3)Poner de manifiesto la existencia de los errores que se suponía que existían o falsar esta hipótesis.

4)Servir de pauta de solución de dichos errores.

5)Incluir los siguientes tipos de acción: recoger información, tomar decisiones, analizar problemas independientes e integrarlos en un único proceso, aumentar los conocimientos -no sólo físicos- del estudiante, integrar materias (Medir y Giralt, 1998).

\section{REFERENCIAS METODOLÓGICAS}

La idea básica nació de la lectura del libro del físico y docente soviético Piotr Kapitsa (1985), en cuyo capítulo IV presenta algunas de las técnicas de trabajo que en los años cincuenta desarrollaba con sus estudiantes, buscando siempre la respuesta a problemas cotidianos más o menos complejos (Él los llamaba sencillos. A juicio del lector curioso, quede el calificativo).
Desde un principio, la idea fue usar una metodología de modelo investigativo tipo APU (1994) o PROPHY (Caillot y Dumas-Carben, 1987), por ejemplo, cuyo esquema podría adecuarse a lo que se pretendía. No se recurrió, en consecuencia, a modelos algorítmicos, sin que ello signifique que en el conjunto de la enseñanza de la asignatura no deba recurrirse a dichos modelos. Algunos, como el de Selvaratnman (1994), son a juicio del autor muy eficaces, sobre todo cuando no se dispone de excesivo tiempo ni medios y hay por delante una prueba tan absurda, pero inevitable, como la selectividad.

No obstante, quedaba fuera de lugar y de tiempo pretender iniciar el trabajo con una investigación experimental en un partido profesional, midiendo tiempos, velocidades, etc., ya sea a pie de campo, ya sobre una filmación o en vídeo. No se descarta en absoluto que pueda tener interés y podría dar lugar a una versión más completa de la experiencia.

Dadas las circunstancias, pues, el modelo de «problema de enunciado abierto» (Gil y Martínez Torregrossa, 1983) podría ajustarse a lo que se buscaba. En este modelo se parte de un problema sin datos numéricos, y su desarrollo implica las siguientes fases:

- análisis cualitativo del problema;

- emisión de hipótesis acerca de los factores que van a influir en la solución;

- diseño de posibles estrategias;

- resolución del problema empezando por el desarrollo algebraico y dejando para el final los datos numéricos extraídos de situaciones reales;

- análisis de los resultados, entendido como una verificación de la consistencia interna.

Este esquema, que a juicio del autor debería aplicarse, con más o menos fidelidad, a la mayoría de procesos de aprendizaje, no sólo de la física sino de la mayoría de ciencias numéricas, parecía el más indicado en principio, no sólo por las implicaciones de tipo cognitivo a que podía conducir (y que en el momento de proponerse la experiencia no interesaban demasiado), sino porque parte de los problemas en apariencia detectados parecían provenir de una enseñanza anterior demasiado volcada en la pura aplicación de algoritmos, entendidos más bien como falsillas, a los problemas.

No podía, no obstante, dejarse de lado la situación de estudiantes de COU, con tiempo limitado por tanto, y con la perspectiva de una prueba determinante en la cual se deberían enfrentar a problemas algorítmicos. Combinar la metodología del modelo investigativo con el esquema de enunciado abierto podía ser una buena solución, y de este modo se hizo.

En resumen, se optó por un esquema de trabajo próximo a los de Gil y Martínez Torregrosa (1983), Furió y 
Guisasola (1993), Varela y Martínez Aznar (1996 y 1997), etc., que combinan el modelo investigativo con el enunciado de tipo abierto.

La diferencia fundamental con el trabajo de estos autores consistía en que aquí, desde un principio, se fijaban variables y se dictaban pautas mínimas de estrategia por parte del profesor como las condiciones en que se desarrollaba el problema, los datos que era preciso recabar y las vías de salida de las situaciones más comprometidas.

No obstante, todavía restaba un amplio abanico de variables a determinar y aproximaciones a realizar, que se dejaba al libre albedrío del alumno.

Otra diferencia fundamental respecto a la metodología citada fue que desde el primer momento se sugirió la posibilidad de realizar todo el cálculo y, en consecuencia, el planteamiento ab origine del problema usando medios automáticos de cálculo, (como por ejemplo una hoja Excel).

\section{ENUNCIADO DEL PROBLEMA}

Un jugador de baloncesto lanza un tiro libre a la canasta desde la posición reglamentaria. Suponiendo fija la velocidad inicial $v_{0}$, suficiente para alcanzar el blanco, determínese el conjunto de ángulos desde los que es posible alcanzar el tanto. Para ello:

- Se supondrá despreciable el rozamiento con el aire, así como que el balón se lanza sin efecto alguno.

- Se considerará enceste si el punto inferior del balón impacta en cualquier punto del diámetro máximo de la canasta, siempre en trayectoria descendente.

- Se considerarán los dos casos: enceste directo y rebote.

- Se supondrá que el jugador lanza desde una altura $h_{0}$.

Resuelta esta parte, ¿es posible determinar el valor mínimo de $\mathrm{v}_{0}$ por debajo del cual es imposible el enceste, no importa bajo que ángulo? (Cuestión voluntaria)

Los datos numéricos (que no se dieron en el enunciado, había que buscarlos, y son los usados por el profesor): $v_{0}=10 \mathrm{~m} / \mathrm{s}, h_{0}=1,80 \mathrm{~m}$. La canasta se encuentra a 3,05 $\mathrm{m}$ de altura, su diámetro es $0,45 \mathrm{~m}$. La personal se lanza a 5,80 $\mathrm{m}$ del fondo del campo. El tablero se sitúa a 1,20 $\mathrm{m}$ del fondo del campo y el aro a $15 \mathrm{~cm}$ del tablero en $\mathrm{su}$ punto más próximo.

\section{MODUS OPERANDI}

Propuesto el enunciado, y aunque en principio se pensó en no dar demasiadas pistas, en la práctica se creyó conveniente fijar las aproximaciones y condiciones de partida, pero siempre dejando lo más abierto posible el problema. Se hizo ver la coherencia de las mismas, aunque lo ideal habría sido no hacerlo, pero el tiempo imponía su ley. Se dejó al arbitrio del alumnado fijar algunos parámetros como la posición exacta de lanzamiento del balón. Quizá sea imprescindible proponer la canasta virtual para el tiro a tablero. También se dejó que fueran los propios alumnos quienes investigasen los datos geométricos del campo, altura de canastas etc. (La experiencia demuestra que el no dárselos es la primera sorpresa ¿Como un problema en que hay que buscar datos?) En cualquier caso, se advirtió que las normas que se daban eran provisionales y que estaba en la decisión de los estudiantes el aceptarlas o no, siempre y cuando se justificara la decisión tomada.

Se dio una velocidad inicial orientativa del balón (en el ejemplo se les propuso unos $10 \mathrm{~m} / \mathrm{s}$ ), pero siempre dejando a su arbitrio aceptarla o no. También se dejaban abiertas:

- la posición inicial del balón, que podría ser, por ejemplo, la propia posición de cada estudiante;

- la mayoría de parámetros iniciales del movimiento;

- la asunción de hipótesis simplificativas;

- la discusión del resultado numérico y, a su vista, de las hipótesis inciales.

Se insistió en que no era un «problema de examen», sino un pequeño trabajo de investigación y como tal debía ser tratado, y que lo importante era un examen exhaustivo de las condiciones y posibles soluciones, así como de las leyes, modelos, simplificaciones y aproximaciones utilizados, más que dar la respuesta exacta que el profesor esperaba.

Se decidió que se trabajara de modo individual, aunque en casa.

\section{El cálculo de la velocidad mínima se dejó como adición voluntaria}

Se dio un plazo de quince días (de viernes a viernes), equivalente a ocho clases, para realizar el trabajo. La experiencia del autor indica que, en general, no deben darse plazos muy largos porque:

- descontextualizan el ejercicio de la marcha normal de la clase;

- en general, no significan más tiempo de trabajo, sino retrasar el comienzo, lo que reincide en el punto anterior;

- las correcciones, revisiones, etc. se dilatan más de lo debido y pierden efectividad.

Los ejercicios se devolvieron corregidos y comentados junto con una copia del mismo trabajo hecho por el profesor. Este sistema de devolver un original junto con 
el trabajo corregido es habitual en la metodología de quien escribe estas líneas y siempre, según su experiencia, de gran efectividad.

El autor mismo impartía dos grupos de COU y, aunque, como se ha dicho reiteradamente, no se pretendía una investigación psicopedagógica sino dar una solución a un problema concreto, en principio se pensó en dejar un grupo como testigo y ensayar la prueba sólo con uno de los dos grupos. Pero una comisión de notables del grupo excluido hizo constar su protesta y prácticamente exigió su derecho a hacer también la prueba en cuestión, lo que venía a demostrar el grado de motivación logrado. Al final se optó por proponer la prueba a ambos grupos, mientras un tercer grupo, en el que impartía otro profesor, quedaba como testigo. Este particular debe tenerse en cuenta a la hora de evaluar los resultados.

\section{PRIMERA EVALUACIÓN}

La solución que se incluye como anexo muestra que el problema puede resolverse dentro de los conocimientos matemáticos de un estudiante como los sujetos de la prueba, puesto que no requiere más que un poco de cálculo diferencial elemental -que incluso puede obviarse- y saber interpretar una ecuación de segundo grado (en este caso con un poco de ingenio, pero que puede substituirse con un poco más de trabajo (Bútikov, Bíkov y Kondrátiev, 1989). Además, tanto el enunciado como la discusión de las soluciones deberían estar al alcance de cualquier estudiante.

De cara al alumno se consideró:

- exposición clara de las condiciones iniciales del problema;

- muestra de las aproximaciones y simplificaciones realizadas, así como de su importancia;

- formulación clara del modelo matemático: sistema referencial y ecuaciones;

- explicación de los pasos que conducen a la solución;

- correcta presentación de los resultados;

- comentarios sobre los mismos, especialmente de cara a su verosimilitud;

- comparación de sus resultados con los hechos experimentales en el propio campo de deporte.

Una vez corregido el trabajo, calificado y comentado, en la primera clase posterior al último día de entrega, se les devolvió junto con una copia del mismo trabajo realizado por el profesor (el que se incluye como anexo). Con base de este documento, y en el propio de cada estudiante, se comentó en clase de forma genérica el trabajo de los alumnos, destacándose los errores más comunes.

Sobre una base de 60 alumnos a los que se propuso el problema se observó inicialmente lo siguiente:
- Más del $50 \%$ no había conseguido datos geométricos correctos, o los había modificado arbitrariamente, en aras de una simplificación matemática del problema, sin entrar a relacionar sus simplificaciones con el hecho de apartarse del resultado: el balón entra (= punto) o no entra (= fallo), y posible jugada para el contrario. Dicho de otro modo, habían seguido fuera de la realidad objetiva y aferrados al problema académico tradicional.

- Un $65 \%$ redujo sus explicaciones a una mera descripción de los pasos matemáticos.

- Sólo un $10 \%$ comentó sus propios resultados.

- Sólo 4 alumnos intentaron hallar la velocidad mínima y lo hacieron dando por supuesto que ésta se logrará para un ángulo de $45^{\circ}$.

- Ni un solo estudiante criticó las hipótesis iniciales.

- Sólo 3 alumnos verificaron la hipótesis de la «trayectoria descendente».

- Ningún estudiante tuvo en cuenta el tamaño del tablero a la hora de plantearse la cuestión del rebote.

- No obstante su presunta base informática y la sugerencia del profesor, un $85 \%$ utilizó métodos exclusivamente «clásicos»y los que emplearon informática lo hicieron para construir gráficos o para redactar el trabajo, no para calcular.

- Algunos (15\%), pese a que se les advirtió, utilizaron fórmulas estereotipadas: alcance máximo, altura máxima, sin caer en la cuenta de que no eran aplicables en este caso.

- Sin embargo, todos plantearon sus ecuaciones tomando como referencia «la posición inicial del balón». Sólo uno ha matizado a qué parte del balón se refería. El resto se quedó en la aproximación del punto, sin decirlo.

- Para un porcentaje bastante alto (35\%), el diámetro del aro no influía en los resultados.

Este resumen demuestra que la intuición original no estaba desencaminada y que la cinemática elemental está muy lejos de ser comprendida ni, mucho menos, asimilada. Aunque es por lo menos aventurado extrapolar un trabajo como éste, con tal número de ítems, su realización debe servir -y para este profesor sirvió- para reflexionar sobre la adecuación de los métodos de enseñanza que se están siguiendo.

\section{REGULACIÓN}

A la vista de estos resultados se pidió a algunos de los estudiantes que los debatieran. Se hizo énfasis especialmente en cuestiones de este estilo: ¿Cómo es posible que el diámetro de la canasta no influya en el resultado? O bien: ¿Por qué es necesario eliminar las soluciones en las que el balón no está en la rama descendente de la 
trayectoria? Las respuestas daban a entender que, a pesar, de todo, seguía firmemente anclada en la mente de los muchachos la idea de que los problemas de física tienen poco que ver con el mundo real. No pasan de ser entelequias.

Fue especialmente difícil convencer a algún estudiante de que afirmaciones como lo he hecho con calculadora no eran un argumento sólido, y lo fue más aún para quienes partieron de las fórmulas estereotipadas de altura o alcance máximos. También se comentaron positivamente las soluciones ingeniosas, correctas o no, destacando su aspecto creativo. De hecho, la clase se convirtió en animado diálogo en que se propusieron mejoras al propio trabajo del profesor.

Algún estudiante preguntó si era posible hallar la distancia real recorrida por el balón. Esta pregunta sirvió de base a otro ejercicio del mismo tipo que no se relaciona para no alargar demasiado el trabajo, pero que aportó soluciones realmente ingeniosas (no hay que olvidar que a aquellas alturas del curso no se manejaba el concepto de integral) en las que se ponía de manifiesto que muchos problemas de física, cuya comprensión e incluso desarrollo y solución están perfectamente al alcance de los alumnos, se quedan en el camino por un recurso excesivo al desarrollo formal matemático (Alonso, 1992)

Hubiera sido altamente útil, pero no se hizo, disponer de alguna filmación y medir directamente sobre ésta. Posteriormente el profesor lo hizo y los resultados fueron altamente congruentes con los encontrados antes, incluso en el caso de que el jugador lanzara el balón con un cierto «picado».

Puede ser una buena práctica pedir a los estudiantes que, con base en el trabajo correcto, elaboren de nuevo el suyo y, si es posible, traten de mejorar el trabajo modelo.

\section{EVALUACIÓN FINAL}

A fin de verificar si se había conseguido o no una mejora en la comprensión-aplicación, se volvió a pasar una prueba semejante a la inicial. Para comparar resultados se pasó la misma prueba. Ninguna de las dos pruebas se había avisado previamente y, en ambas, se permitió el uso de material auxiliar: libros de teoría o apuntes.

Se realizó en ambas pruebas un test de igualdad de medias sobre una base de distribución normal, aceptable dado el número de ítems. Hay que insitir en el hecho de que, en el grupo de control, se daba además la variable «distinto profesor» o, si se prefiere, «distinta metodología global», lo cual puede falsear los resultados al atribuir a una sola variable lo que debería atribuirse por lo menos a dos. Para minimizar esta posibilidad, los ejercicios de ambas pruebas se redactaron en común por los dos profesores.
Los resultados estadísticos de ambas pruebas, la inicial y la de control, se exponen a continuación en el cuadro I. Ambos ejercicios están calificados sobre 10 .

Los resultados estadísticos parecen indicar lo siguiente:

- Los tres grupos pueden considerarse como pertenecientes a una misma población, no hay diferencias significativas a un nivel de 0,05.

- Después del ejercicio, los grupos A y B han mejorado significativamente, en tanto que la mejora del grupo $\mathrm{C}$ no puede considerarse como significativa a nivel 0,65 .

- La comparación entre los grupos A, B y C en la segunda prueba permite concluir que la diferencia de las medias entre los grupos A y B no es significativa a nivel 0,05 , en tanto que la diferencia de qualesquiera de éstos con el grupo $\mathrm{C}$ es significativa tanto a nivel 0,05 como 0,01 .

\section{CONCLUSIONES}

Comparando los resultados con los objetivos propuestos y considerando el desarrollo completo de la experiencia, cabe decir que aquéllos se lograron, incluso los englobados en el epígrafe 5, puesto que los estudiantes debieron cubrir todos y cada uno de los puntos allí indicados, aunque no fuera éste el objetivo principal.

- Quedaban claros los papeles de los parámetros fundamentales: velocidad, posición y ángulos iniciales. La trayectoria, al menos en el modelo newtoniano, está determinada desde el principio, precisamente por estos parámetros.

- Era evidente que la gravedad no cambiaba de dirección a partir del punto más alto. El gráfico de la trayectoria, continua y derivable desde el punto de vista matemático, lo dejaba claro.

- Las «fórmulas estereotipadas» para calcular el alcance máximo, la altura máxima, etc. no funcionaban, puesto que no eran expresión adecuada del modelo buscado.

- Las aproximaciones que implicaba el modelo estaban todas determinadas por la solución final: «balón entra o no entra». La comparación con el modelo real parecía indicar que estaban bien aplicadas.

- El modo de resolver el problema no era único, aunque las soluciones permitían, como además indica la experiencia, un margen de tolerancia muy pequeño.

- Existía una solución no algorítmica, la que cada día resuelven los jugadores de baloncesto al lanzar sus tiros libres. A esta solución se llegaba, no a través de ecuaciones y fórmulas, sino mediante otra técnica básica en el trabajo experimental: el ensayo y error y la experiencia acumulada, que permitía en breves segundos un cálculo intuitivo de ángulo y velocidades iniciales. 


\begin{tabular}{|l|c|c|c|c|c|c|}
\cline { 2 - 7 } \multicolumn{1}{c|}{} & \multicolumn{2}{c}{ Prueba inicial } & \multicolumn{2}{c|}{ Prueba de control } \\
\cline { 2 - 8 } & A & B & C & A & B & C \\
\hline Alumnos & 28 & 27 & 32 & 30 & 5,28 & 5,45 \\
\hline Media & 4,05 & 3,97 & 3,75 & 1,36 & 1,48 & 4,15 \\
\hline Desviación & 1,27 & 1,33 & 1,45 & 1,98 \\
\hline
\end{tabular}

Ensayo de medias

\begin{tabular}{|l|c|c|c|c|c|c|}
\hline$Z_{(\mathrm{A}-\mathrm{B})}$ & 0,23 & $-1,96<\mathrm{Z}<1,96$ & $\mathrm{Z}_{\left(\mathrm{A}^{\prime}-\mathrm{B}^{\prime}\right)}$ & $-0,45$ & $-1,96<\mathrm{Z}<1,96$ & $\mathrm{Z}>2,58$ \\
\hline $\mathrm{Z}_{(\mathrm{A}-\mathrm{C})}$ & 0,85 & $-1,96<\mathrm{Z}<1,96$ & $\mathrm{Z}_{\left(\mathrm{A}^{\prime}-\mathrm{C}^{\prime}\right)}$ & 2,58 & $\mathrm{Z}>2,58$ & \\
\hline $\mathrm{Z}_{(\mathrm{B}-\mathrm{C})}$ & 0,61 & $-1,96<\mathrm{Z}<1,96$ & $\mathrm{Z}_{\left(\mathrm{B}^{\prime}-\mathrm{C}^{\prime}\right)}$ & 2,84 & & \\
\hline $\mathrm{Z}_{\left(\mathrm{A}-\mathrm{A}^{\prime}\right)}$ & $-3,56$ & $\mathrm{Z}<-2,58$ & & & & \\
\hline $\mathrm{Z}_{\left(\mathrm{B}-\mathrm{B}^{\prime}\right)}$ & $-3,90$ & $\mathrm{Z}<-2,58$ & & & & \\
\hline $\mathrm{Z}_{\left(\mathrm{C}-\mathrm{C}^{\prime}\right)}$ & $-0,90$ & $-1,96<\mathrm{Z}<1,96$ & & & \\
\hline
\end{tabular}

Esta experiencia puede además ser una buena base para analizar la incertidumbre de los resultados, salvada la gran dificultad que implica evaluar de modo exacto la velocidad y el ángulo de lanzamiento y, en menor grado, el punto exacto de lanzamiento. Todas estas variables, dado lo ajustado de los resultados, son muy importantes.

Finalmente, parece haber buenas razones para considerar que esta experiencia es una buena herramienta para mejorar la comprensión y la aplicación práctica de la cinemática, detectando y corrigiendo conceptos equivocados:

1) Constituye un elemento altamente motivador.

2) Ayuda a imbricar la física en la vida diaria.

3) Dado lo ajustado de las soluciones, obliga a trabajar con cuidado y esmero y a meditar muy seriamente la asunción de aproximaciones. Dicho de otra forma, pone de manifiesto la complementariedad y la no-oposición entre lo cualitativo y lo cuantitativo.

4) Destaca el papel de las condiciones iniciales del proble$m a$, identifica la trayectoria y pone de manifiesto la utilidad-inutilidad de muchas de las fórmulas estereotipadas.

5)Permite la incoporación de nuevas tecnologías a la enseñanza de la física, poniendo de manifiesto sus ventajas.
6) Dentro de la misma línea son posibles otros experimentos relacionados con otros deportes como el fútbol, el alpinismo o el patinaje, aunque el ejemplo elegido sea el más realista posible con el mínimo número de variables (sólo tres, en realidad: velocidad, posición y ángulo iniciales). Dadas las velocidades que se usan, la hipótesis «despreciando rozamientos» es perfectamente adecuada.

7) Permite, dentro de ciertos límites, un comprobación experimental de resultados o, al menos, su aplicación definitiva: «Se marca tanto o no».

En cuanto al aparato matemático, en apariencia complicado, se pone en evidencia la necesidad de un buen manejo del álgebra y la trigonometría elementales, y exige la solución, con un poco de ingenio, de ecuaciones en apariencia complicadas, especialmente al buscar la velocidad mínima. Al mismo tiempo, sin embargo, se ha verificado que los nuevos instrumentos, como la hoja electrónica de cálculo, de manejo sencillo y eficaz, permiten hallar rápida y exactamente una solución que, con los métodos tradicionales, exigiría cálculos mucho más complejos.

Aunque los resultados inmediatos conseguidos no sean excelentes, el tipo de trabajo planteado parece una buena herramienta para que los propios alumnos detecten sus malos hábitos, errores y conceptos equivocados, y los corrijan. 


\section{REFERENCIAS BILIOGRÁFICAS}

ALONSO, M. (1992). Entrevista con. Revista Española de Física, 6(3), pp. 36-38.

APU (Assessment of Performance Unit) (1994). Science report for teachers. Association for Science Education. Londres: Hertfordshire.

BÚTIKOV, E., BÍKOV, A. y KONDRÁTIEV. A. (1989). Física en ejemplos y problemas. Moscú: Mir.

CAILLOT, M. y DUMAS-CARRÉ, A. (1987). Prophy: un enseignement d'une méthodologie de résolution de problèmes en mathématiques et en physique. Rapports de Recherches, 12, pp. 199-244.

FERNÁNDEZ RAÑADA, A. et al. (1994). Física básica. Madrid: Alianza Editorial.

FURIÓ, C. y GUISASOLA, J. (1993). ¿Puede ayudar la historia de la ciencia a comprender por qué los estudiantes no comprenden los conceptos de carga potencial eléctricos? Revista Española de Física, 7(3), pp. 46-50.

GIL, D. y MARTÍNEZ-TORREGROSA, J.A. (1983). A model for problem solving in accordance with scientific methodology. European Journal of Science Education, 5(4), pp. 447-455.

GUTIÉRREZ, J. (1993). Enseñanza de la física, un reto a la imaginación. Revista Española de Física,7(3), pp. 50-53.

KAPITSA, P. (1985). Experimento, teoría y práctica. Moscú: Mir.

MARTÍNEZ-AZNAR, M.M. y VARELA NIETO, P. (1996). De la resolución de problemas al cambio conceptual. Investigación en la escuela, 28, pp. 59-68.

MARTÍNEZ PONS, J.A. (1995). Cinemática. Revista Zeus, 24, pp. 16-20.

MEDIR, M. y GIRALT, F. (1998). Programa APQUA. Documentación. Barcelona: Reverté.

SELVARATNMAN (1994). Science report for teachers. Association for Science Education. Londres: Hertfordshire.

VARELA, P. y MARTÍNEZ-AZNAR, M.M.(1997). Investigar y aprender resolviendo problemas abiertos de física. Revista Española de Física, 11(12), pp. 32-37.

[Artículo recibido en noviembre de 1996 y aceptado en mayo de 1999.] 


\section{ANEXO}

\section{SOLUCIONES}

Se proponen al lector estas soluciones como las más adecuadas al nivel en que se plantea el problema.

\section{DETERMINACIÓN DE LOS ÁNGULOS}

La figura da una representación esquemática del problema que, dadas las condiciones iniciales, puede reducirse a dos dimensiones.

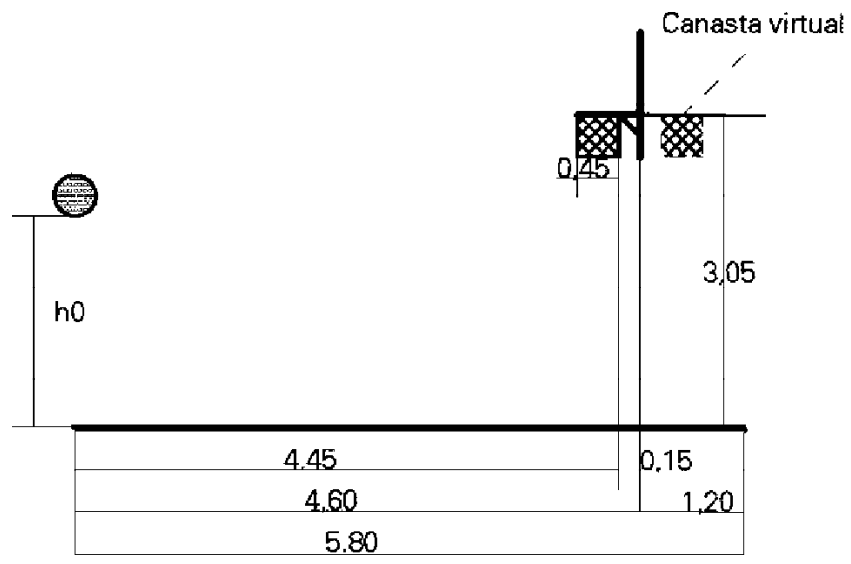

Por simplicidad del cálculo se va a tomar el origen del sistema coordenado justo en el polo del balón, con lo cual la altura de la canasta será $h=3,05-h 0$.

Se distinguirán dos casos:

a) tiro directo, tiro a canasta;

b) rebotado, tiro a tablero, en este caso por obvias consideraciones geométricas, el problema equivaldrá a encestar en una canasta virtual, imagen especular, respecto al tablero de la canasta real. El espesor del tablero no influye en el resultado, puesto que se trabaja con la imagen geométrica de la canasta.

\section{TIRO A CANASTA}

Las ecuaciones de posición y velocidad del balón en el sistema de referencia elegido serán

$$
\begin{array}{ll}
x=v_{0} t \cos \alpha ; & v_{x}=v_{0} \cos \alpha \\
y=v_{0} t \operatorname{sen} \alpha-\frac{g t^{2}}{2} ; & v_{y}=v_{0} \operatorname{sen} \alpha-g t
\end{array}
$$

donde $\alpha$ es el ángulo de lanzamiento.

El problema tiene una infinidad de soluciones. Se busca el valor mínimo de modo que la pelota impacte en el punto más alejado del aro.

Para la velocidad inicial dada, las ecuaciones de las trayectorias, referidas al punto de lanzamiento serían

$$
y=x \tan \alpha-4,9 x^{2} /\left(v_{0} \cos \alpha\right)^{2}
$$

Hay que calcular las posibles trayectorias para cada uno de los extremos del aro. Se trata de imponer, por tanto, que la trayectoria pase por los puntos $\mathrm{A}(4 ; 1,25) ; \mathrm{B}(4,45 ; 1,25)$; operando se obtienen dos posibles familias de trayectorias.

La solución se ha obtenido con la opción Solver. Puede también obtenerse por tanteo, llegando a 29,67 ; sin embargo, observando los valores o la gráfica, se ve que la primera de la trayectorias alcanza A en su fase ascendente, pero no así la última. Habrá que 
encontrar el valor a partir del cual se alcanza el aro en la fase descendente, para ello y por medio de Solver se calcula la altura máxima imponiendo las restricciones de que esté entre los datos, y que $x$ esté entre 4 y 4,45, encontrándose, en consecuencia, que serán válidas las trayectorias que cumplan $29,80^{\circ}>\alpha>29,81^{\circ}$. Si $76,02^{\circ}>\alpha>77,55^{\circ}$ En ésta se cumplen todas las condiciones.

\section{TIRO A TABLERO}

Ahora se trata de imponer el paso por los puntos A' $(4,75 ; 1,25)$ y B' $(5,20 ; 1,25)$.

\section{Trayectorias}

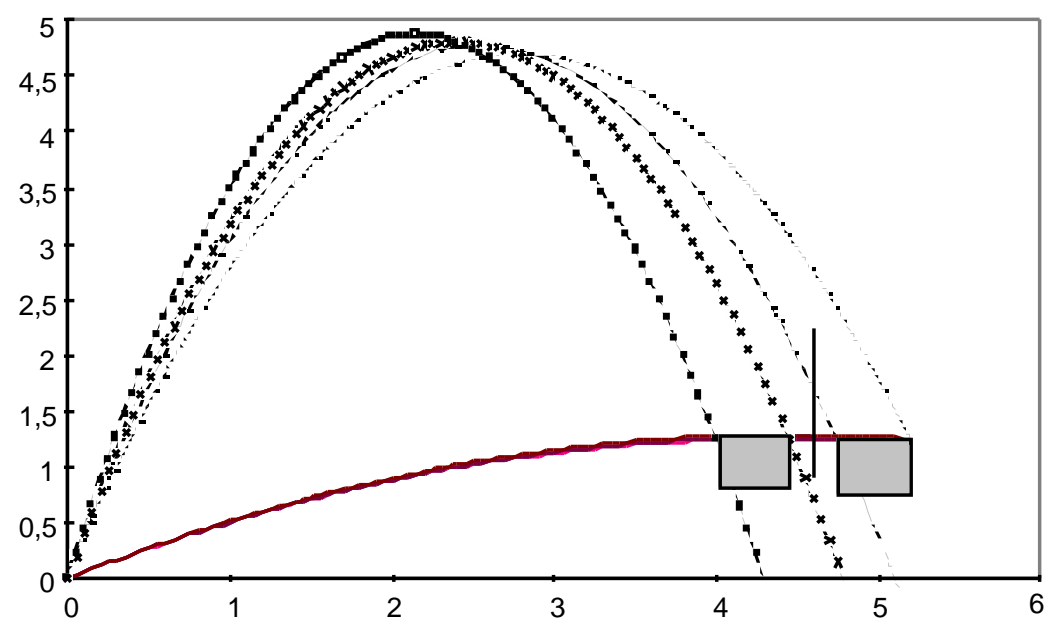

El cálculo da ahora las trayectorias que cumplan:

$29,77^{\circ}<\alpha<30,17^{\circ}$. Se cumplen todas las restricciones, luego la familia es válida.

$74,97^{\circ}>\alpha>73,35$ : Ahí si se cumplen las condiciones; sin embargo, hay que comprobar que todas ellas dan en el tablero, para ello se calcula la altura correspondiente al último ángulo y para un valor de $x$ de 4,60 $\mathrm{m}$.

El resultado es de 2,75 m, evidentemente exagerado. Suponiendo ahora un tablero que alcanza $2 \mathrm{~m}$, se vuelve a plantear la ecuación, obteniéndose que el borde del tablero se alcanza para un ángulo de $74,60^{\circ}$, lo que limita los ángulos aceptables.

Concretando, se llega a las conclusiones siguientes, fijadas las condiciones de velocidad inicial y de altura de lanzamiento; son posibles los siguientes ángulos:

En tiro a canasta: $\quad 28,80^{\circ}<\alpha<28,81^{\circ}$. (Prácticamente inexistente)

$$
76,02^{\circ}<\alpha<77,55^{\circ}
$$

En tiro a tablero: $\quad 29,77^{\circ}<\alpha<30,17^{\circ}$

$$
74,97^{\circ}>\alpha>74,60^{\circ}
$$

Obsérvese el escaso margen de maniobra que dejan los cálculos. Los jugadores los realizan por pura intuición, gracias a sus condiciones naturales y a la repetición de lanzamientos, que les permiten un índice de aciertos muy elevado. 


\section{CUESTIÓN VOLUNTARIA: DETERMINACIÓN DE LA VELOCIDAD MÍNIMA}

Se trata de encontrar, si existe, una velocidad mínima por debajo de la cual no es posible alcanzar el aro, sea cual sea el ángulo.

El problema es bastante complejo y se tratará de resolver de un modo simple.

\section{Solución algebraica}

En principio, el problema va a resolverse apuntando al extremo del diámetro del aro más próximo al lanzador.

Puesto que para cualquier velocidad inicial, en general, existirán dos trayectorias, una de las cuales, al menos, corresponderá al tiro por elevación, de momento se prescindirá de la restricción «rama de caída» que, si es necesario, se introducirá más adelante.

Vaya de entrada que la solución «El punto es el más alto de la trayectoria.» no es correcta; basta para ello considerar que el alcance máximo sobre la horizontal no corresponde al punto de altura máxima.

Pártase de la ecuación de trayectoria, obtenida de eliminar el tiempo entre las ecuaciones de posición. Para un determinado valor de $x$ e $y$ depende de dos variables, ángulo y velocidad iniciales.

Podría intentarse una solución a partir de la teoría de funciones, pero, entre otras razones, por estar fuera del alcance matemático de los estudiantes a quienes va dirigido, se usará otro método, si cabe incluso con mayor contenido físico. Para ello se va a reescribir la ecuación de la trayectoria del modo siguiente:

$$
\begin{aligned}
& g x^{2} \tan ^{2} \alpha-2 v_{0} x \tan \alpha+g x^{2}+2 v_{0}^{2} y=0 \Rightarrow \\
& \tan \alpha=\frac{\left.v_{0}^{2} \pm \sqrt{v_{0}^{4}-g\left(g x^{2}+2\right.} v_{0}^{2} y\right)}{g x}
\end{aligned}
$$

Sólo tienen sentido físico las soluciones reales para $\tan \alpha$, lo que quiere decir que el discriminante debe ser no negativo:

$$
\mathrm{v}_{0}^{4}-\left(2 \mathrm{gyv}_{\mathrm{o}}^{2}+\mathrm{g}^{2} \mathrm{~s}^{2}\right) \geq 0
$$

Dada la forma matemática de esta expresión, es evidente que el valor mínimo de la velocidad inicial corresponderá a la igualdad.

Resolviendo la ecuación bicuadrada en $v_{0}$ se obtiene como única solución aceptable físicamente:

$$
\left.v_{0, \min }=\sqrt{g\left(y+\sqrt{y^{2}+x^{2}}\right)}=\sqrt{9,8\left(1,25+\sqrt{1,25^{2}+4^{2}}\right.}\right)=7,30 m s^{-1}
$$

Con este valor se encuentra que el único ángulo posible con el que se impactaría justo en el aro es $53,52^{\circ}$; es decir, que la velocidad mínima para lograr encestar es $7,30 \mathrm{~ms}$ y el enceste se logrará con un ángulo de $53,52^{\circ}$.

Solución informática

Para esta segunda opción existe una solución utilizando la potencialidad de la hoja Excel. Para ello se creará una columna con los tiempos en función de un $d t$ arbitrario. Se establecerá una velocidad inicial arbitraria, en este caso se ha ubicado en F2.

Se establecerá también un ángulo inicial arbitrario y se programarán X e Y del modo tradicional.

Se definirá una nueva casilla en que se calcule $v_{0}$ en función de una $x$ cualquiera y su tiempo.

Usando Solver se pedirá que esta función se minimice cambiando las celdas donde se encuentra el ángulo, el incremento de tiempo y la propia velocidad inicial que se introdujo al principio. Este proceder es necesario por las propias limitaciones de Excel.

Como restricción se impondrá que un par de casillas cualesquiera, pero homólogas, tomen los valores de las coordenadas del punto del aro previsto.

Los resultados que se obtienen son bastante realistas. Sin embargo, la hipótesis de considerar enceste si el polo inferior del balón cae sobre el aro puede pecar de optimista. Más seguridad se tendría si se aceptara enceste seguro sin que ninguna parte del balón tocara el aro. Dado que el diámetro del balón reglamentario de baloncesto es de 20 a $22 \mathrm{~cm}$, el problema equivaldría a encestar en un aro de unos $23 \mathrm{~cm}$ concéntrico con el real.

Replanteado el problema en estos términos, los resultados que se obtienen manteniendo las mismas condiciones iniciales restantes son:

$$
29,74^{\circ}>\alpha>29,67^{\circ} ; \quad 76,36^{\circ}>\alpha>77,21^{\circ} ; \quad 29,83^{\circ}<\alpha<30,06^{\circ} ; \quad 74,62^{\circ}>\alpha>73,71^{\circ}
$$

Haciendo las mismas consideraciones que en los resultados obtenidos inicialmente, se observa que las trayectorias correspondientes a la primera familia de ángulos son todas ascendentes. En consecuencia, debe eliminarse esta solución y, aceptando el mismo valor para el tablero, la última familia queda en:

$$
74,60^{\circ}>\alpha>73,71^{\circ}
$$

Con estas nuevas hipótesis, la velocidad mínima valdrá $v_{\min }=7,406 \mathrm{~m} / \mathrm{s}$ con un ángulo de $57,03^{\circ}$

Un detalle final es que un jugador de baloncesto medianamente entrenado, por simple intuición y con un buen adiestramiento y práctica, es capaz en breves segundos de calcular distancias y velocidad, así como de coordinar sus movimientos de modo que logre el enceste en un elevado porcentaje de casos, a pesar de la poca tolerancia que parece desprenderse de los resultados. 